1999

\title{
Investigation of the Thickness Mode in Surface Stabilized Ferroelectric Liquid Crystal Cells
}

\author{
O.E. Kalinovskaya \\ Trinity College Dublin, Ireland \\ J.K. Vij \\ Trinity College Dublin, Ireland \\ Yu. V. Tretyakov \\ Trinity College Dublin, Ireland
}

See next page for additional authors

Follow this and additional works at: https://arrow.tudublin.ie/engscheleart2

Part of the Electrical and Computer Engineering Commons

\section{Recommended Citation \\ Kalinovskaya, O.E. et al. (1999). Investigation of the thickness mode in surface stabilized ferroelectric liquid crystal cells. Liquid Crystals vol. 26, no. 5, pg. 717- 722.}

This Article is brought to you for free and open access by the School of Electrical and Electronic Engineering at ARROW@TU Dublin. It has been accepted for inclusion in Articles by an authorized administrator of ARROW@TU Dublin. For more information, please contact arrow.admin@tudublin.ie, aisling.coyne@tudublin.ie, gerard.connolly@tudublin.ie.

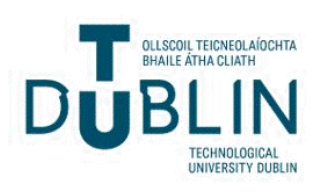


Authors

O.E. Kalinovskaya, J.K. Vij, Yu. V. Tretyakov, Yuri Panarin, and Yu. P. Kalmykov

This article is available at ARROW@TU Dublin: https://arrow.tudublin.ie/engscheleart2/142 


\title{
Investigation of the thickness mode in surface stabilized ferroelectric liquid crystal cells
}

\author{
O. E. KALINOVSKAYA, J. K. VIJ*, YU. V. TRETYAKOV, YU. P. PANARIN \\ Department of Electronic Engineering, Trinity College, University of Dublin, \\ Dublin 2, Ireland
}

and YU. P. KALMYKOV

Institute of Radio Engineering and Electronics of the Russian Academy of Science, Vvedenskogo Sq. 1, Fryazino, Moscow 141120, Russia

(Received 14 August 1998; in final form 2 November 1998; accepted 17 December 1998)

\begin{abstract}
Dielectric properties of surface stabilized ferroelectric liquid crystal (SSFLC) cells have been investigated both experimentally and theoretically. The thickness mode is found to consist of two relaxation processes. A decomposition of an analytical solution of the complex dielectric permittivity $\varepsilon(\omega)$ in terms of these processes is given. The experimental results show agreement with theory and lead to the conclusion that the thickness mode can be separated into two processes. It has been found that the ratio of the dielectric strengths for the two processes depends on the cell thickness, and that the ratio of their frequencies is approximately equal to 10. The lower frequency process is assigned to the relaxation within the bulk and the higher frequency process is assigned to the fluctuations of molecules at the two surfaces of the cell. The latter process is seen only in thicker cells for the reason that anchoring at surfaces predominantly controls the behaviour of thinner cells.
\end{abstract}

\section{Introduction}

Dielectric spectroscopy is a powerful technique for investigating the relaxation mechanism and thereby the modes of operation of ferroelectric liquid crystal (FLC) cells [1-3]. For frequencies below $1 \mathrm{MHz}$, two collective relaxation processes are known to exist: one is a fluctuation in the tilt angle $(\theta)$-soft mode; the second is a fluctuation in the azimuthal angle $(\varphi)$-Goldstone mode. The dielectric properties of FLC cells are found to be dependent on the cell thickness [4]. In sufficiently thick FLC cells the dielectric properties are governed by the helical structure; the dielectric strength and the relaxation time of the Goldstone mode depend on the helical pitch [5]. A theory for the dielectric response of a helical cell in the $\mathrm{SmC}^{*}$ and the $\mathrm{SmA}$ phase has been developed by Carlsson et al. [5] and Žekš et al. [6]. In a surface stabilized ferroelectric liquid crystal (SSFLC) cell the helix is unwound by the surface interactions, and the dielectric parameters of the Goldstone mode depend on the cell thickness. We recently developed a theoretical model [4] to explain the thickness dependence of the dielectric behaviour for SSFLC cells. This model is based on the dynamic distribution of the azimuthal angle $\varphi$ of the director with respect to the $X$-axis, shown in figure 1 ,

*Author for correspondence for a chevron cell. The external electric field, $E_{0} \cos \omega t$, is applied along the $X$-axis.

The spatial and temporal torque balances are described by the dynamic equation

$$
\gamma_{\varphi} \sin ^{2} \theta \frac{\partial \varphi}{\partial t}=K_{\varphi} \sin ^{2} \theta \frac{\partial^{2} \varphi}{\partial x^{2}}+\mathbf{P}_{\mathrm{s}} E_{0} \cos \omega t \sin \varphi .
$$

Equation (1) is solved for $\varphi$ subject to the boundary conditions detailed in [4]. The complex permittivity is found in terms of the surface anchoring parameters, the elastic constant and the cell thickness. The equations for the director profiles, in terms of the variation of the azimuthal angle, as a function of $X$ for both the bookshelf and chevron cell geometries of the cell have been determined. The relaxation mode resulting from these calculations is called the $X$-mode or the thickness mode, to distinguish it from the helical dynamic process. The dielectric measurements in certain cases have shown the existence of two relaxation processes in a SSFLC cell. Under certain conditions the 'thickness' mode was shown not to follow the Debye behaviour.

In this paper, we wish to investigate the dependence of the relaxation mode/modes on the sample thickness. We analyse the analytical solution of the dynamic equation for the thickness mode in two relaxation processes, Debye 

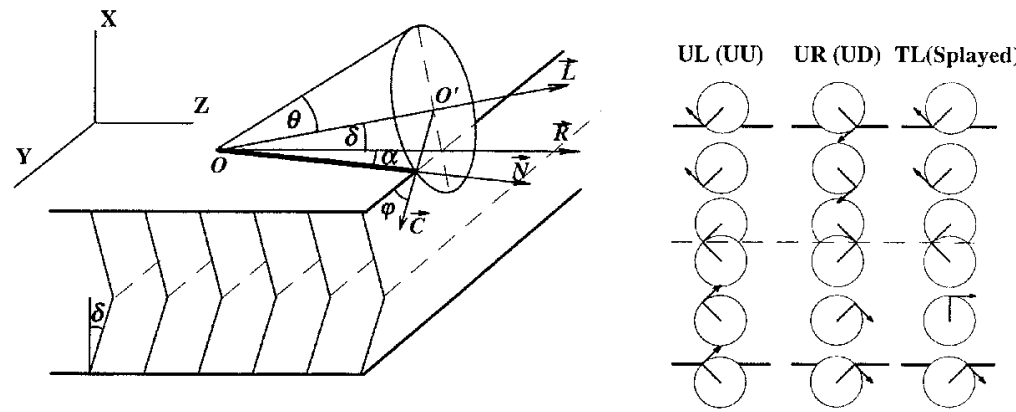

Figure 1. The smectic layer structure for the chevron geometry in FLC cells. $\mathbf{N}=$ molecular director, $\mathbf{L}=$ smectic layer normal, $\mathbf{R}=$ rubbing direction, $\mathbf{C}=C$ director, $\theta=$ molecular tilt angle, $\varphi=$ azimuthal angle, $\delta=$ smectic layer tilt. On the right, director profiles: $\mathrm{UL}=$ uniform left, $\mathrm{UU}=$ uniform up, $\mathrm{UR}=$ uniform right, $\mathrm{UD}=$ uniform down, $\mathrm{TL}=$ twisted left.

and Cole-Davidson [7], and examine their relative strengths. The nature of these processes is investigated for cells, possessing the chevron structure, as these possess higher thermodynamic stability compared with their bookshelf counterparts.

\section{Theory}

The normalized complex dielectric permittivity of a chevron SSFLC cell, based on the model outlined in the introduction and detailed in our previous paper [4], is found to be:

$$
\begin{aligned}
\varepsilon(\omega)= & \frac{2(1+2 \Lambda)}{\left(-\mathrm{i} \omega \tau_{\mathrm{c}}\right)^{1 / 2}} \\
& \times \frac{\left[1-\cos \left(-\mathrm{i} \omega \tau_{\mathrm{c}}\right)^{1 / 2}\right]}{\left[\sin \left(-\mathrm{i} \omega \tau_{\mathrm{c}}\right)^{1 / 2}+2 \Lambda\left(-\mathrm{i} \omega \tau_{\mathrm{c}}\right)^{1 / 2} \cos \left(-\mathrm{i} \omega \tau_{\mathrm{c}}\right)^{1 / 2}\right]}
\end{aligned}
$$

where

$$
\tau_{\mathrm{c}}=\gamma d^{2} /(4 K) .
$$

$\Lambda=K / d w$ is a dimensionless parameter, $d$ is the thickness of the cell, $K$ is the elastic constant, $\gamma$ is the rotational viscosity, $w$ is the surface anchoring energy and $\tau_{\mathrm{c}}$ is a relaxation time for $X$ mode/modes for a chevron cell. In the derivation of equation (2), the electronic and atomic polarizations were neglected and the complex dielectric permittivity was also normalized, with $\varepsilon_{\mathrm{s}}=1, \varepsilon_{\infty}=0$.

Since equation (2) shows a complicated dependence of complex permittivity on frequency, we therefore analyse equation (2) by first fitting the dielectric data generated by this equation to known relaxation functions for different values of $\Lambda$ using a WinFit (Novocontrol) program. The best results of fitting are obtained if the data generated by equation (2) are fitted to the following function [10]:

$$
\varepsilon(\omega)=\frac{\Delta \varepsilon_{1}}{1+\mathrm{i} \omega \tau_{1}}+\frac{\Delta \varepsilon_{2}}{\left[1+\left(\mathrm{i} \omega \tau_{2}\right)^{\alpha}\right]^{\beta}}
$$

with $\alpha \cong 1$. Equation (4) is the sum of relaxation processes given by the Debye term and for $\alpha=1$, the ColeDavidson term. Results of numerical fitting are shown in figures 2 and 3. Figure 2 shows the normalized dielectric strength whereas figure 3 shows the relaxation times for the two processes obtained using this fitting

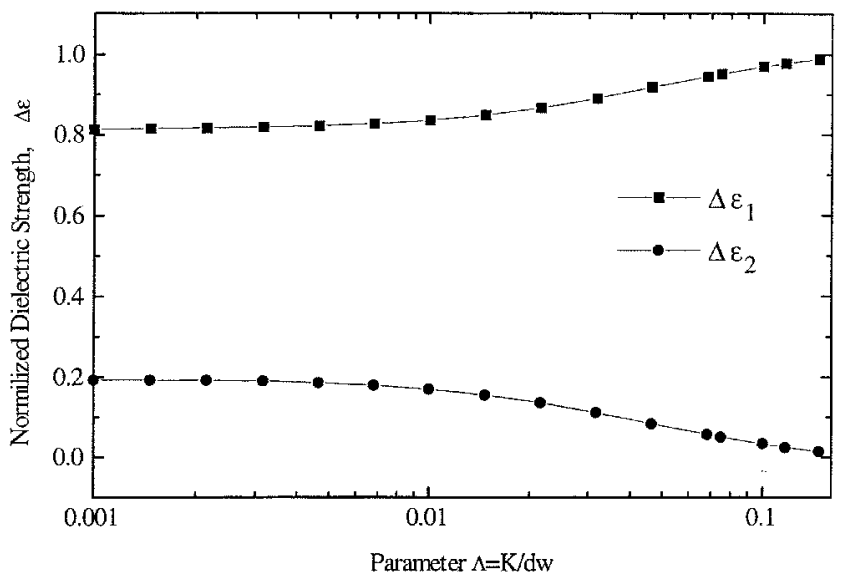

Figure 2. Plot of normalized dielectric strengths as a function of the parameter $\Lambda$.

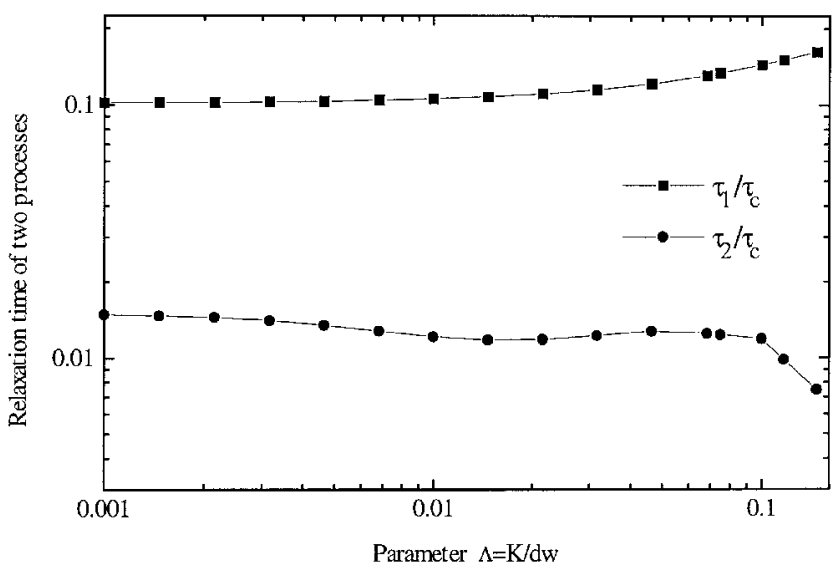

Figure 3. Relaxation times as a function of the parameter $\Lambda$. 
procedure. It should be noted that the parameter $\beta$ found from the data fitting depends on $\Lambda$.

Figure 2 shows that when the parameter $\Lambda$ is large enough (i.e., much greater than 0.01 ), there exists predominantly only a single relaxation process in the thickness mode of a SSFLC cell, since $\Delta \varepsilon_{2}$ tends to zero as $\Lambda \gg 0.01$. This single relaxation process appears to follow the Debye equation. However for small values of $\Lambda$, two relaxation processes are found in the thickness mode of the SSFLC cells. Hence the existence of the second process depends on the parameter $\Lambda$, and indirectly on the thickness of the cell, since the latter is the only variable parameter in the simulation.

To analyse the spectrum of the complex dielectric permittivity $e(\omega)=\varepsilon^{\prime}(\omega)-\mathrm{i} \varepsilon^{\prime \prime}(\omega)$ generated by equation (2), we introduce the parameters $\eta$ and $\xi$ defined as in [8]:

$$
\begin{aligned}
& \xi=\frac{\tau_{0} \omega\left[\varepsilon^{\prime}(\omega)-\varepsilon_{\infty}\right]}{\varepsilon^{\prime \prime}(\omega)} \\
& \eta=\frac{\varepsilon_{\mathrm{S}}-\varepsilon^{\prime \prime}(\omega)}{\tau_{0} \omega \varepsilon^{\prime \prime}(\omega)}
\end{aligned}
$$

where $\varepsilon_{\mathrm{s}}=\lim _{\omega \rightarrow 0} \varepsilon(\omega), \varepsilon_{\infty}=\lim _{\omega \rightarrow \infty} \varepsilon(\omega), \tau_{0}=\mathrm{a}$ time constant which is of the order of the relaxation time of the system under investigations. $\xi=\tau_{0} / \tau, \eta=\tau / \tau_{0}$ when the spectrum $\varepsilon(\omega)$ consists of only a single Debye process:

$$
\varepsilon(\omega)=\varepsilon_{\infty}+\frac{\Delta \varepsilon}{1+\mathrm{i} \omega \tau} .
$$

It was shown by Barriol et al. [8] that if the spectrum $\varepsilon(\omega)$ is a sum of two Debye processes, namely:

$$
\varepsilon(\omega)=\varepsilon_{\infty}+\frac{\Delta \varepsilon_{1}}{1+\mathrm{i} \omega \tau_{1}}+\frac{\Delta \varepsilon_{2}}{1+\mathrm{i} \omega \tau_{2}}
$$

then the plot of variable $\eta$ versus $\xi$ is a straight line with $\omega$ as the running variable:

$$
\eta=\frac{\tau_{1}+\tau_{2}}{\tau_{0}}-\frac{\tau_{1} \tau_{2}}{\tau_{0}^{2}} \xi .
$$

In all other cases, Salefran [9] found that the plot of $\eta$ versus $\xi$ is no longer a straight line, but is a curve with frequency as a variable. For a single Debye relaxation, we get a single point on the $\eta$ versus $\xi$ plot; a curve may lead to the system having either more than two processes or one or both of these processes may have a distribution of relaxation times.

For the analysis of a chevron cell, we use the aforesaid transformation equations (5) and (6). Figure 4 shows the dependence of $\eta$ on $\xi$ and figure 5 shows dielectric loss $\varepsilon^{\prime \prime}(\omega)$ versus $\omega \tau_{\mathrm{c}} /(2 \pi)$ on a log-log plot. Plots in figures 4 and 5 were calculated using equation (2) with the parameter $\Lambda$ equal to (i) 0.001 and (ii) 0.1 .

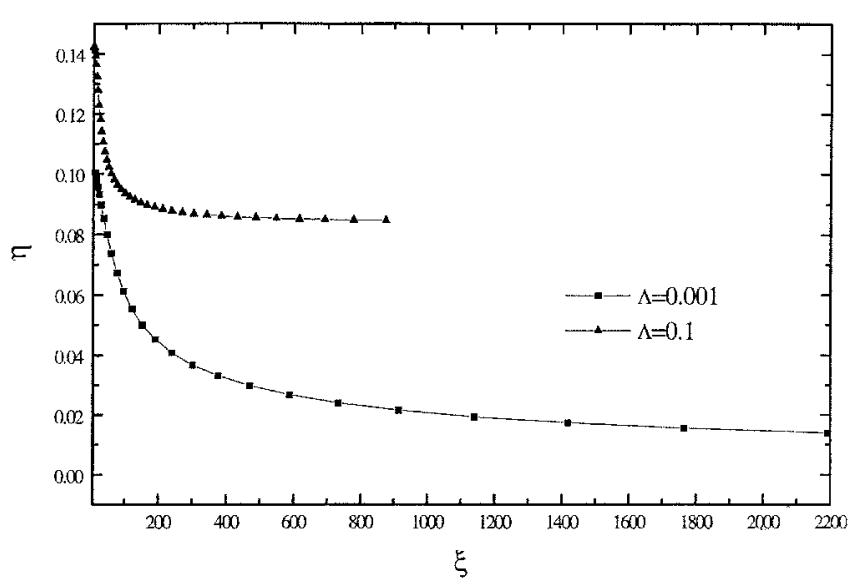

Figure 4. Plot of parameter $\eta$ versus parameter $\xi$ for two different values of $\Lambda$.

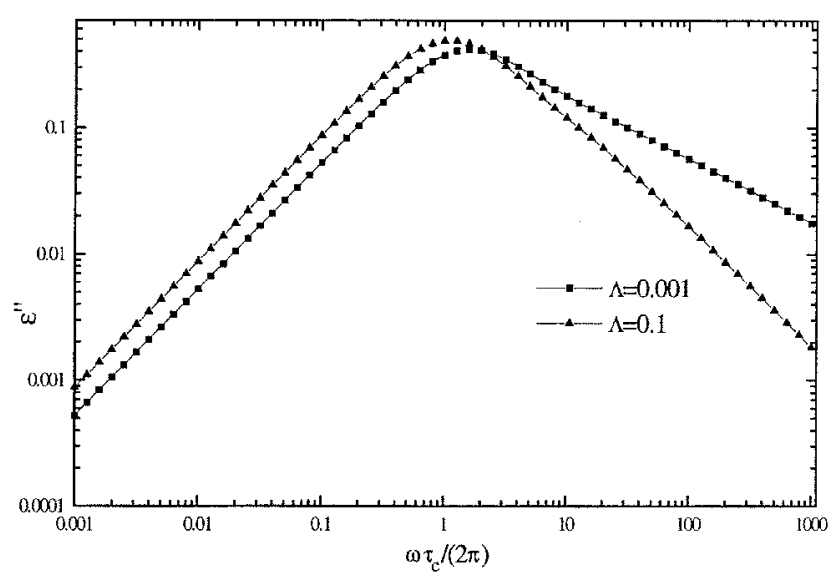

Figure 5. Theoretical dielectric loss spectra for FELIX 18/100 for values of parameter $\Lambda=0.1$ and $\Lambda=0.001$.

From the plot in figure 4 and for $\Lambda=0.001$ and $\Lambda=0.1$, we note that the plots are curves other than straight lines and these curves are also far removed from a single point. On applying the physical concepts of Selfran [9], we find that the thickness mode consists of two processes which cannot be approximated by a sum of two single Debye processes. As mentioned above, the system may consist of more than two Debye-type processes or two relaxations times with a distribution around one or both of these processes. However, by analysing curves in figures 4 and 5 in the low frequency region, we note that the main relaxation process is Debye-type and the second process in this frequency range can also be approximated by the Debye equation in the low frequency range. For $\xi<200, \eta$ versus $\xi$ is a straight line whereas for higher values of $\xi$ the slope changes. Also, in the high frequency region the slope of the curve in figure 5 is less than predicted for a single Debye process. 
This type of behaviour at higher frequencies can best be described by a Cole-Davidson [7] type process written in the form:

$$
\varepsilon(\omega)=\varepsilon_{\infty}+\frac{\Delta \varepsilon}{(1+\mathrm{i} \omega \tau)^{\beta}}
$$

where $0<\beta<1$. In the low frequency region, ColeDavidson behaviour approximates to a Debye process with a slope equal to unity, whereas for high frequencies, the slope is less than unity and is equal to $\beta$. Based on this analysis we can infer that the dielectric response of a SSFLC cell is the sum of the Debye and Cole-Davidson processes:

$$
\varepsilon(\omega)=\varepsilon_{\infty}+\frac{\Delta \varepsilon_{1}}{1+\mathrm{i} \omega \tau_{1}}+\frac{\Delta \varepsilon_{2}}{\left(1+\mathrm{i} \omega \tau_{2}\right)^{\beta}}
$$

where $\varepsilon_{\mathrm{s}}=\Delta \varepsilon_{1}+\Delta \varepsilon_{2}=1, \varepsilon_{\infty}=0$.

Another conclusion that can be obtained from figure 2 is that for $\Lambda=0.1$, the second process has very low dielectric strength relative to the first, and the relaxation time in comparison to the main Debye process is different from that for $\Lambda=0.001$. Since the second process has relatively low dielectric strength for $\Lambda=0.1$, it may not in any case be easy to find this process. This leads to the conclusion that the presence of the second process in the thickness mode of a SSFLC cell depends on the parameter $\Lambda$ (or the cell thickness).

The objective of this paper is to estimate the dielectric parameters for both processes based on the model and then to find these processes experimentally. The estimation of the dielectric strength and the relaxation time of both processes is based on the transformations given by equations (5) and (6). The parameter $\beta$ can be found from the analysis of the slope of the dielectric peak in the high frequency region of figure 5 for an appropriate value of $\Lambda$. For $\omega \tau_{2} \ll 1$, we can expand equation (11) and on taking the first two terms in the series expansion, the dielectric response of a SSFLC becomes:

$$
\varepsilon \cong \frac{\Delta \varepsilon_{1}}{1+\mathrm{i} \omega \tau_{1}}+\frac{\Delta \varepsilon_{2}}{1+\mathrm{i} \omega \beta \tau_{2}}
$$

The parameters $\Delta \varepsilon_{1}, \Delta \varepsilon_{2}, \tau_{1}, \beta \tau_{2}$ of equation (11) are estimated using equations (5), (6) and (9), as shown below. By substituting $\varepsilon(\omega)$ from equation (2) into equations (5) and (6) and comparing the Taylor series expansions for $\eta$ and $\xi$ in the low frequency range, we obtain the dependence $\eta=A-B \xi$. Then by using (9) we get the relations for the parameters of both processes:

$$
\begin{gathered}
\frac{\tau_{1}+\beta \tau_{2}}{\tau_{\mathrm{c}}}=A=\frac{29+250 \Lambda-1428 \Lambda^{2}-12584 \Lambda^{3}}{30(1+2 \Lambda)\left(9+12 \Lambda-460 \Lambda^{2}\right)} \\
\frac{\tau_{1} \beta \tau_{2}}{\tau_{\mathrm{c}}^{2}}=B=\frac{1+17 \Lambda-228 \Lambda^{2}-3400 \Lambda^{3}}{180(1+\Lambda)\left(9+12 \Lambda-460 \Lambda^{2}\right)} .
\end{gathered}
$$

From equations (13) and (14), the relaxation time $\tau_{1}$ of the main Debye process and the value of $\beta \tau_{2}$ for the second high frequency process in the thickness mode of a chevron cell are found to be as follows:

$$
\begin{gathered}
\frac{\tau_{1}}{\tau_{\mathrm{c}}}=\frac{A}{2}+\left(\frac{A^{2}}{4}-B\right)^{1 / 2} \\
\frac{\beta \tau_{2}}{\tau_{\mathrm{c}}}=\frac{A}{2}-\left(\frac{A^{2}}{4}-B\right)^{1 / 2} .
\end{gathered}
$$

Using values of $\tau_{1}$ and $\beta \tau_{2}$ from equations (15) and (16), the dielectric strengths of both processes can be evaluated using (12) and the results become:

$$
\Delta \varepsilon_{1}=\frac{\left(Q-\tau_{2} / \tau_{\mathrm{c}}\right) \tau_{\mathrm{c}}}{\tau_{1}-\tau_{2}} \quad \Delta \varepsilon_{2}=\frac{\left(\tau_{1} / \tau_{\mathrm{c}}-Q\right) \tau_{\mathrm{c}}}{\tau_{1}-\tau_{2}} .
$$

where $Q=(1+10 \Lambda) / 12(1+\Lambda)$.

\section{Results and discussion}

The FLC mixture used was FELIX 18/100 purchased from Hoechst, Germany. This FLC mixture is selected because of its relatively large helical pitch of about $25 \mu \mathrm{m}$, such that the fabrication of surface stabilized FLC cells with sufficiently large thicknesses is possible. The measurements are made on homogeneously aligned cells with 6,15 and $30 \mu \mathrm{m}$ spacings at room temperature. Dielectric spectra were fitted by using WINFIT (Novocontrol) program up to $n=3$ processes as described by the Havriliak and Negami equation [10]:

$$
\varepsilon(\omega)=\frac{G}{\omega^{1-S} C_{0}}+\sum_{j=1}^{n} \frac{\Delta \varepsilon_{j}}{\left[1+\left(\mathrm{i} \omega \tau_{j}\right)^{\alpha_{j}}\right]^{\beta_{j}}}
$$

where $G$ is the d.c. conductivity, $S$ is a fitting parameter usually close to zero and $C_{0}$ is a geometrical capacitance. Figures 6, 7 and 8 show measured dielectric loss spectra and their fit to the spectra for the chevron cells under investigation. From these figures we find that for cells with thicknesses of 30 and $15 \mu \mathrm{m}$, the thickness mode (or $X$-mode) consists of two processes: the main low frequency process is Debye type and the second high frequency process is of Cole-Davidson type, as predicted by the theory. But for the cell with a thickness of $6 \mu \mathrm{m}$, only one (Debye type) process appears in the spectra. We showed in our earlier paper [4] that the dielectric 


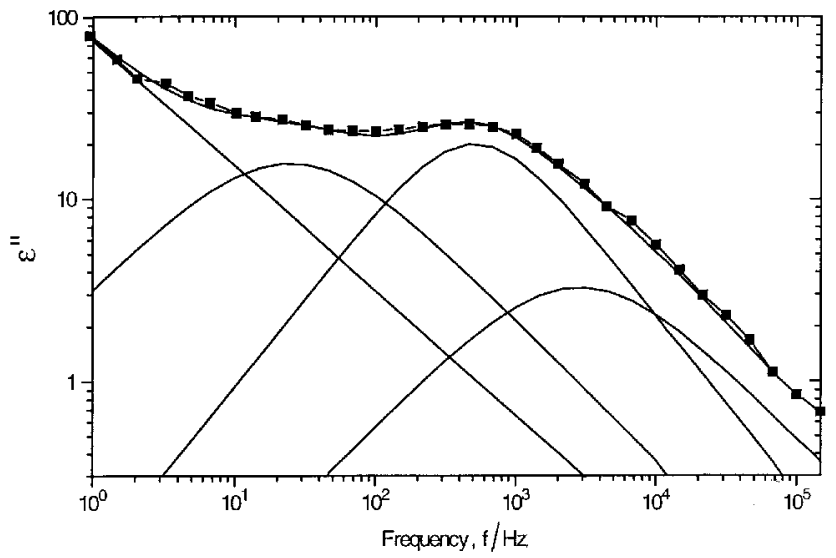

Figure 6. Experimental dielectric loss spectra for FELIX $18 / 100$ (Hoechst) for a cell of thickness $30 \mu \mathrm{m}$.

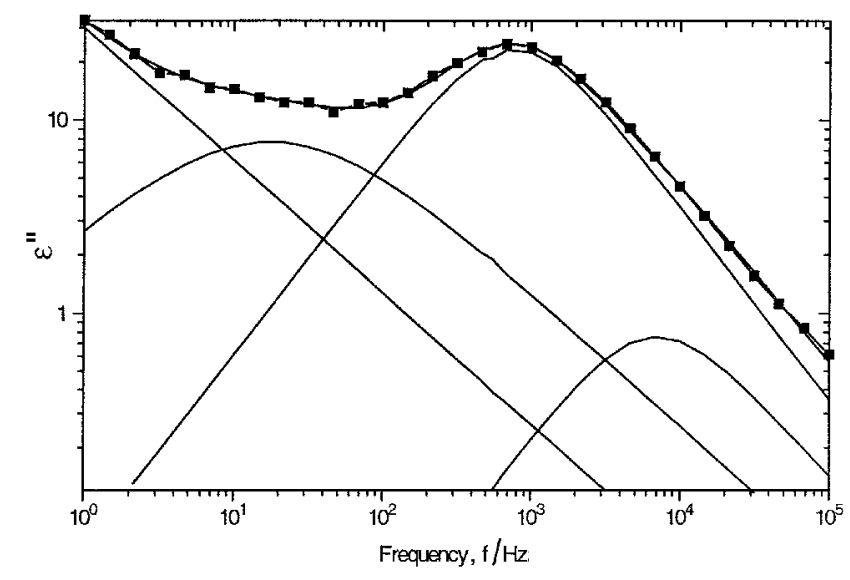

Figure 7. Experimental dielectric loss spectra for FELIX $18 / 100$ (Hoechst) for a cell of thickness $15 \mu \mathrm{m}$.

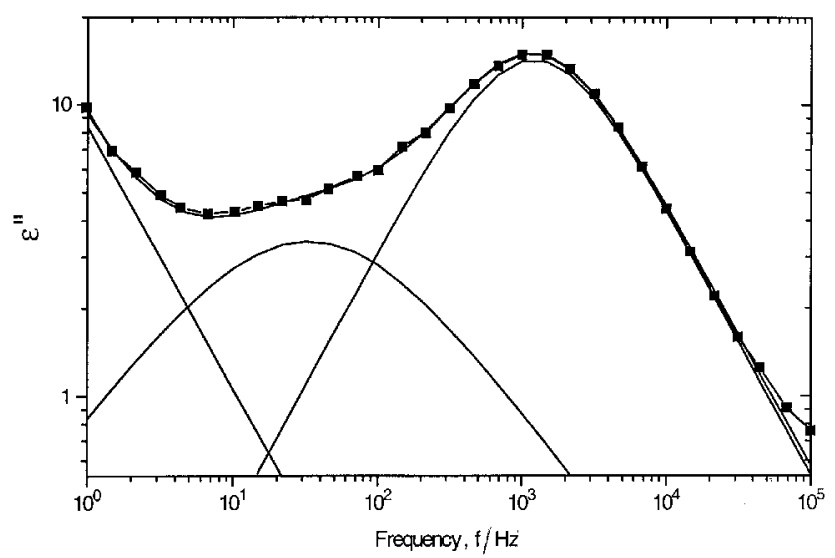

Figure 8. Experimental dielectric loss spectra for FELIX $18 / 100$ (Hoechst) for a cell of thickness $6 \mu \mathrm{m}$.

parameters of the $X$-mode are found to be very strongly dependent on the parameter $\Lambda=K / d w$ or the cell thickness, provided this is the only variable in the experiment. A change in the cell thickness influences both constituent components of the $X$-mode. These are being confirmed by the experimental results. Decreasing the cell thickness $d$, and consequently increasing $\Lambda$, the high frequency process is suppressed. It has indeed been found that for a $6 \mu \mathrm{m}$ cell, the high frequency process is absent. For a $30 \mu \mathrm{m}$ cell, we find $\varepsilon_{1} / \varepsilon_{2}=5$ and $\tau_{1} / \tau_{2}=6$ and for a $15 \mu \mathrm{m}$ cell $\varepsilon_{1} / \varepsilon_{2}=28$ and $\tau_{1} / \tau_{2}=8$. These results are in qualitative agreement with theory.

Figures 5, 6 and 7 show the presence of a relatively very low frequency peak centred at $\sim 30 \mathrm{~Hz}$. This presumably is due to a domain process which is commonly observed $[11,12]$ at very low frequencies in FLC cells.

Next, the problem of assignment of the relaxation processes is considered. Equation (2) is based on the calculation of the dynamic variation of the azimuthal angle of the director, called the director profile, as a function of $X$. Figure 9 shows such a plot of the static variations of the director profile. The dynamic profiles correspond to the fluctuations of the static profile with time. For thicker cells, the profile can fluctuate both at the surfaces as well as in the bulk simply for the reason that the azimuthal angle is found to be greatly dependent on $X$ in comparison with that for a thin cell. However for thinner cells, the director profile seemingly can only fluctuate within the bulk since in this case the azimuthal angle does not appear to depend much on $X$. Here the surface anchoring significantly influences the fluctuations within the bulk too; such a significant control of the surface anchoring is lost as the cell thickness is gradually increased. Hence process 1 given by the relaxation time $\tau_{1}$, common to both thick and thin cells, belongs to the

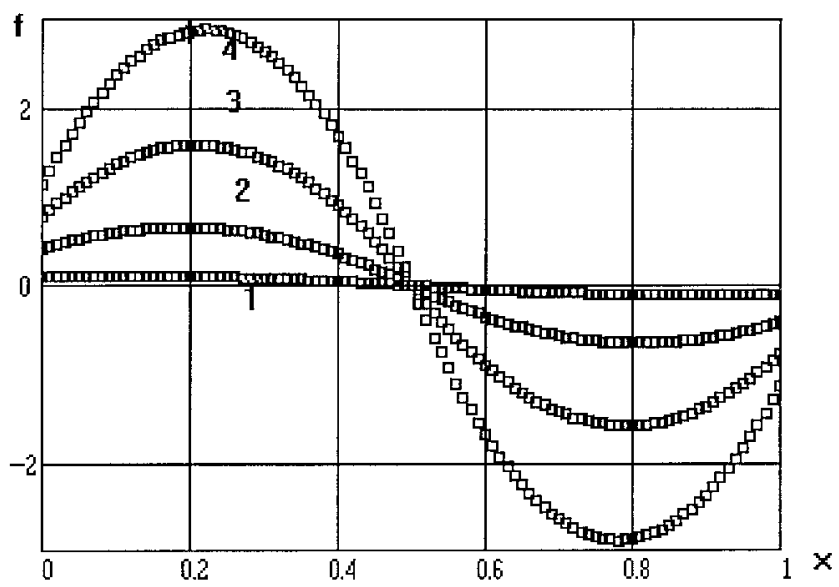

Figure 9. The static distribution of the azimuthal angle $\varphi(x)$, computed from the analytical solution of equation (1), about its equilibrium position $\varphi=\varphi_{0}$ for a cell possessing chevron geometry, the function $f=\varphi(x)-\varphi_{0}$; the basic parameters are $d=10^{-6} \mathrm{~m}, E=10^{-4} \mathrm{~V} \mathrm{~m}^{-1}, K=10^{-11} \mathrm{~J} \mathrm{~m}^{-3}$, $w=10^{-4} \mathrm{~J} \mathrm{~m}^{-2}, \varphi_{0}=60^{\circ}$. On the figure, curves are numbered $1-4$ in the order of cell thickness: $1,3,5$ and $7 \mu \mathrm{m}$. 
fluctuations within the bulk, whereas $\tau_{2}$ seen only, in the thicker cells, belongs to fluctuations at the surfaces. From another point of view, director fluctuations at the surfaces and the bulk are unresolvable in a thin cell, unlike the situation in a relatively thick cell.

\section{Conclusion}

Qualitative agreement between predictions from our model and experimental results show that cells with large sample thicknesses exhibit more than a single relaxation time. The results show two relaxation modes for the parameter $\Lambda(\Lambda=K / d w)<0.01$, whereas almost a single relaxation mode is found for $\Lambda \gg 0.01$. The results also show that the observed single relaxation mode is of the Debye type. The thicker cells show a complicated frequency dependence of the complex permittivity and this behaviour has been resolved in terms of the two modes. In a thick cell, the low frequency mode is of Debye type, whereas the high frequency mode is of ColeDavidson type. The higher frequency mode appears to arise from the director fluctuations at the surfaces of the cell; a variation in the microscopic properties of surfaces gives rise to a macroscopic fluctuation or a distribution in the relaxation times, and consequently to a ColeDavidson type mechanism. On the contrary, the low frequency mode seen even in a thin cell is assigned to the fluctuations in the bulk similar to that in a thick cell. The higher frequency mode relates to the fluctuations of the director at the surfaces. The results of the dielectric experiments indirectly support our calculations for the director profiles in FLC cells, given in figure 9, which are also supported by measurements of Fuzi and Sambles [13] using guided modes technique.

The authors thank the European Commission for funding this work through the INTAS-96-1411 grant.

\section{References}

[1] Gouda, F., Skarp, K., and Lagerwall, S. T., 1991, Ferroelectrics, 113, 165.

[2] Pavel, J., Glogarova, M., and Bawa, S. S., 1987, Ferroelectrics, 67, 221.

[3] Kremer, F., Schönfeld, A., Vallerien, S. U., Kapitza, A., Zentel, R., and Fischer, E. W., 1990, Phys. Rev. A, 42, 3667.

[4] Panarin, Yu. P., Kalmykov, Yu. P., Maclughadha, S. T., XU, H., and VIJ, J. K., 1994, Phys. Rev. E, 50, 4763.

[5] Carlsson, T., Žekš, B., Filipiě, C., and Levstik, A., 1990, Phys. Rev. A, 42, 877.

[6] Žekš, B., Carlsson, T., Musevič, I., and KutnjaKURbanc, B., 1993, Liq. Cryst., 15, 103.

[7] Davidson, D. W., and Cole, R. H., 1950, J. Chem. Phys., 18, 1417.

[8] Barriol, J., Boule, P., and Diguet, R., 1969, Compt. rend. Acad. Sci. (Paris), 268C, 1977.

[9] Salefran, J. L., 1977, Chem. Phys. Lett., 45, 124.

[10] Havriliak, S. JR., and Negami, S., 1967, Polymer, 8, 161.

[11] Panarin, Yu. P., Xu, H., Maclughadha, S. T., and VIJ, J. K., 1994, Jpn. J. appl. Phys., 33, 2648.

[12] HaVriliak, S. JR., ViJ, J. K., and Ni, M., 1999, Liq. Cryst., 26, 3.

[13] Fuzi, Y., and Sambles, J. R., 1993, Liq. Cryst., 13, 1. 